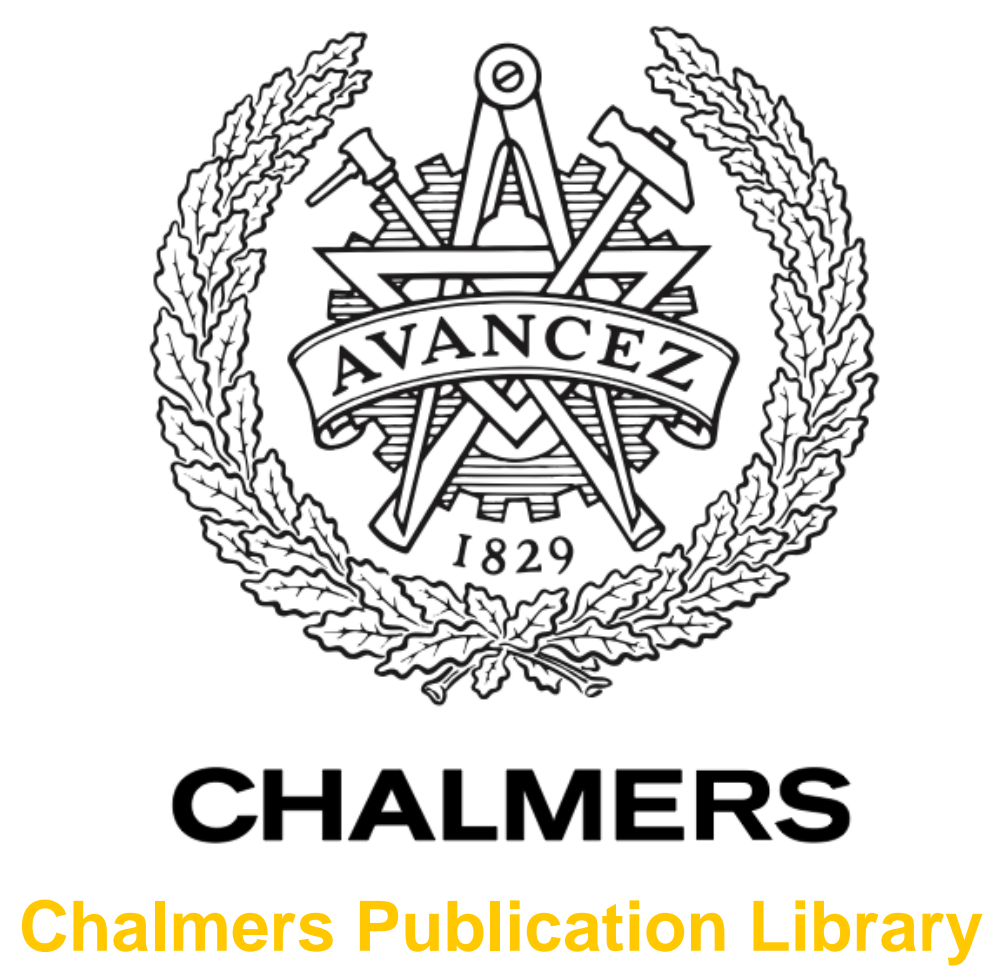

Capacity analysis of multibeam joint decoding over composite satellite channels

This document has been downloaded from Chalmers Publication Library (CPL). It is the author's version of a work that was accepted for publication in:

45th Asilomar Conference on Signals, Systems and Computers, ASILOMAR 2011;Pacific Grove, CA;6 November 2011through9 November 2011 (ISSN: 10586393)

Citation for the published paper:

Christopoulos, D. ; Chatzinotas, S. ; Matthaiou, M. (2011) "Capacity analysis of multibeam joint decoding over composite satellite channels". 45th Asilomar Conference on Signals, Systems and Computers, ASILOMAR 2011;Pacific Grove, CA;6 November 2011through9 November 2011 pp. 1795-1799.

http://dx.doi.org/10.1109/ACSSC.2011.6190331

Downloaded from: http://publications.lib.chalmers.se/publication/158633

Notice: Changes introduced as a result of publishing processes such as copy-editing and formatting may not be reflected in this document. For a definitive version of this work, please refer to the published source. Please note that access to the published version might require a subscription. 


\title{
Capacity Analysis of Multibeam Joint Decoding over Composite Satellite Channels
}

\author{
Dimitrios Christopoulos*, Symeon Chatzinotas*, Michail Matthaiou ${ }^{\dagger}$, and Björn Ottersten* ${ }^{*}$ \\ ${ }^{*}$ SnT - securityandtrust.lu, University of Luxembourg \\ email: \{dimitrios.christopoulos, symeon.chatzinotas, bjorn.ottersten\}@uni.lu \\ ${ }^{\dagger}$ Chalmers University of Technology, Gothenburg, Sweden, email: michail.matthaiou@chalmers.se \\ ${ }^{\ddagger}$ Royal Institute of Technology (KTH), Sweden, email: bjorn.ottersten@ee.kth.se
}

\begin{abstract}
The throughput of current multibeam satellite systems is limited by self interference. Interference mitigation techniques have the potential to significantly increase the spectral efficiency of these satellite communication systems. The present contribution investigates the ergodic capacity of the return link of a multibeam satellite system, where full frequency reuse is employed and user signals are jointly processed at the gateway. The proposed model incorporates correlated satellite antennas over Rician channels which represent some inherent characteristics of satellite communications. Additionally, the effects of shadowing caused by user mobility, are modeled via the lognormal distribution. Hence, a composite Rician/lognormal fading channel with fully correlated receive antennas is considered. For this channel, a new lower bound on the ergodic capacity is analytically deduced and verified through simulations.
\end{abstract}

\section{INTRODUCTION}

Next generation satellite communication (SatCom) networks are striving for terabit capacity [1]. Considering the return link of these systems, great challenges arise with the provision of high throughput, interactive services to small and energy efficient, mobile terminals. Novel interference mitigation techniques that have been recently applied to terrestrial networks [2] are expected to provide a new tool for the design of future SatComs. In the present contribution, the main objective is to determine the potential capacity gains of the uplink of a multibeam satellite system, that jointly decodes mobile users' signals. On this basis, a closed-form lower bound on the ergodic capacity of the considered system is derived. The tightness of the bound is examined across a wide Signal-toNoise (SNR) range. Moreover, the proposed bound facilitates the investigation of the impact of the model parameters on the channel capacity. To the end of providing a link between theoretical work and real system implementation, a realistic satellite link budget is described so that system throughput can be calculated from the channel ergodic capacity. Subsequently, throughput results demonstrate the potential gains of multiuser decoding in the uplink of multibeam SatComs when compared with the performance of conventional four color schemes.

The rest of the present paper is structured as follows. A brief review of the existing related work is provided in Section II. The considered channel model is described in Section III. Section IV explores the theoretical capacity of the described channel, including the analytical lower bound calculations and the generated numerical results. Conclusions are drawn in
Section V, along with future extensions of the present work.

\section{RELATED WORK}

The capacity of multiple-input multiple-output (MIMO) systems over independent identically distributed (i.i.d.) Gaussian channels was first calculated by [3] . When multiple users are jointly processed by a hyper-receiver then a MIMO Multiple Access Channel (MIMO MAC) is realized and multi-user detection (MUD) is performed. Over the last decade, extensive research in terrestrial cellular systems employing MUD techniques has demonstrated that substantial capacity gains can be obtained [4]-[14]. To the best of the authors' knowledge, the first results on the capacity of a joint-processing (i.e. jointly decoding the received signals) system can be found in [4]. In [7], multipath fading is additionally considered where it is proven to be beneficial. A further extension of the above to account for the effect of Line-of-Sight (LoS) components can be found in [8]. Next, lognormal shadowing was incorporated by the authors of [9]. Also, in the recent work of [10], tight lower capacity bounds for composite Rayleigh-lognormal MIMO fading channels were derived. Finally, several other fading distributions have been considered, such as the composite Nakagami- $m /$ lognormal [12] and Nakagami- $m$ /gamma fading models [11], [15].

Albeit the extensive literature for terrestrial networks, little is known about the performance of multibeam joint decoding techniques in SatComs. In this direction, the work of [13] elaborated on the uplink capacity of a multibeam satellite system. The ergodic capacity of MIMO satellite channels was also investigated by [14]. Furthermore, the authors in [16] used tools of random matrix theory to upper bound the ergodic capacity and compute the outage probability of a MIMO Land Mobile Satellite system (LMS). Lastly, a multiuser decoding algorithm was presented in [17].

While these prior works have significantly improved our knowledge on the ergodic capacity characterization of SatCom systems, some important issues remain unsolved. In this light, a multibeam joint decoding system suitable for SatCom is hereafter considered. The underlying fading model is inherently general since it is a combination of small-scale Rician and large-scale lognormal fading. The latter manifestation is typically caused due to user mobility. Additionally, the proposed model includes an accurate approximation of the multi- 
beam antenna radiation pattern. Similar channel realizations have been considered in [18]-[20]. Under these conditions, a new lower bound on the ergodic capacity is proposed that becomes exact at high SNRs and offers useful insights into the implications of the model parameters.

Notation: Throughout the paper, $\mathcal{E}[\cdot],(\cdot)^{T},(\cdot)^{\dagger}, \odot$ and $\otimes$ denote the expectation, the transpose and the conjugate transpose of a matrix, as well as the Hadamard and the Kronecker product between matrices, respectively. $\mathbf{I}_{n}$ denotes an identity matrix of size $n, \mathbb{I}_{n \times m}$ a $n \times m$ matrix full of ones, $\mathbf{1}_{n}$ a $n$ dimensional vector of ones and $\mathbf{0}_{n \times m}$ a $n \times m$ matrix full of zeros, respectively. Moreover, $\mathbf{X}_{d}=\operatorname{diag}(\mathbf{x})$ is a diagonal matrix composed of the elements of vector $\mathbf{x}=\left[x_{1}, x_{2}, \ldots, x_{n}\right]$. A random matrix denoted as $\mathbf{G}_{n \times m} \sim \mathcal{C N}\left(0, \mathbf{I}_{n}\right)$ is composed of i.i.d. complex elements. Each complex element, denoted as $\mathbf{g}_{i j} \sim \mathcal{C N}(0,1)$, has i.i.d. real and imaginary components each distributed as $\mathcal{N}(0,1 / \sqrt{2})$.

\section{Channel Model}

Let us consider a multibeam satellite scenario for mobile terminals. The focus is on the uplink (i.e. the link from the users to the satellite) while the feeder link (i.e. the link from the satellite to the earth gateway station) is assumed ideal. More specifically, we consider a cluster of $n$ spot-beams covering $n$ user terminals, uniformly distributed in the service area, each equipped with a single antenna. A single user is scheduled to transmit per beam during a specific time slot. The input-output expression for the $i$-th beam reads as

$$
y_{i}=\sum_{j=1}^{n} z_{i j} x_{j}+v_{i}
$$

where $z_{i j}$ is the complex channel coefficient between the $i$-th beam and the $j$-th user and $v_{i}$ is the additive white Gaussian noise (AWGN) measured at the receive antenna. To the end of investigating the uplink satellite channel the following characteristics will be incorporated in the channel model: beam gain $b_{i j}$, lognormal shadowing $\xi_{j}$, Rician fading $h_{i j}$ and antenna correlation. Hence, (1) can be reformulated:

$$
y_{i}=\sum_{j=1}^{n} b_{i j} h_{i j} \xi_{j} x_{j}+v_{i} .
$$

Shadowing $\xi_{j}$ only depends on the $j^{\text {th }}$ user position as a result of the practical collocation of the satellite antennas ${ }^{1}$. Following from (2), the general baseband channel model for all beams in vectorial form is given by

$$
\mathbf{y}=\mathbf{Z x}+\mathbf{v},
$$

where $\mathbf{y}, \mathbf{x}$ and $\mathbf{v}$ are $n \times 1$ receive, transmit, and noise vectors respectively. Channel matrix $\mathbf{Z}_{n \times n}$

$$
\mathbf{Z}=\mathbf{B} \odot \mathbf{H}_{R} \mathbf{\Xi}_{d}^{1 / 2} .
$$

Note that all matrices are square $n \times n$, while the matrix $\mathbf{B}$ models the satellite antenna gain. It is composed of the square

\footnotetext{
${ }^{1}$ Each user sees all the satellite antennas under the same elevation angle.
}

roots of the gain coefficients calculated using the well accepted method of Bessel functions [21]

$$
g_{i j}\left(\theta_{i j}\right)=G_{\max }\left(\frac{J_{1}(u)}{2 u}+36 \frac{J_{3}(u)}{u^{3}}\right)^{2}
$$

where $u=2.07123 \sin \theta_{i j} / \sin \theta_{3 d B}$, and $J_{1}, J_{3}$ are the first kind Bessel functions, of order one and three respectively. The $j$-th user corresponds to an off-axis angle $\theta_{i j}$ with respect to the boresight of the $i$-th beam where $\theta_{i}=0^{\circ}$. Beam gain for each satellite antenna-user pair, depends on the spotbeam antenna pattern and on the user position. Assuming that the positioning of every user remains constant in the duration of a codeword, $\mathbf{B}$ reduces to a deterministic real positive matrix, composed of the square roots of the aforementioned coefficients, that models the position dependent beam gain. Each user-receiver antenna pair has a different gain due to the antenna radiation pattern, hence $\mathbf{B}$ is multiplied elementwise (i.e. Hadamard product) with the small-scale, fading matrix $\mathbf{H}_{R}$. The latter consists of random i.i.d. nonzero mean Gaussian elements and models the Rician fading component as follows [13]:

$$
\mathbf{H}_{R}=\sqrt{\frac{K}{K+1}} \overline{\mathbf{H}}_{R}+\sqrt{\frac{1}{K+1}} \tilde{\mathbf{H}}_{R},
$$

where $K$ is the Rician factor, $\overline{\mathbf{H}}_{R}$ is a deterministic unit rank matrix modeling the LoS signal component and $\tilde{\mathbf{H}}_{R}$ is a complex random matrix representing the scattered components.

The effects of antenna correlation are considered using the Kronecker correlation model [22]. Hence, a realization of $\tilde{\mathbf{H}}_{\mathbf{R}}$ is generated according to

$$
\tilde{\mathbf{H}}_{R}=\mathbf{R}_{r}^{1 / 2} \mathbf{H}_{w} \mathbf{R}_{t}^{1 / 2},
$$

where $\mathbf{H}_{w} \sim \mathcal{C N}\left(0, \mathbf{I}_{n}\right)$. An inherent characteristic of SatCom is the high correlation among the received signals. Total absence of scatterers on the satellite side, the practical colocation of the receive antennae due to on-board limitations and the long propagation path render the receive signals highly correlated. Hence, unit rank receive correlation is assumed, such that $\mathbf{R}_{r}^{1 / 2}=\mathbb{I}_{n \times n}$. On the other hand, the effects of correlation can be ignored on the transmit side; this implies that $\mathbf{R}_{t}^{1 / 2}=\mathbf{I}_{n}$. As a result, the Rayleigh component of the total channel matrix is

$$
\tilde{\mathbf{H}}_{R}=\mathbb{I}_{n} \mathbf{H}_{w} \mathbf{I}_{n}=\mathbb{I}_{n} \mathbf{H}_{w} .
$$

As far as the large-scale fading matrix $\boldsymbol{\Xi}_{d}$ is concerned, we first note that its entries are modeled via the classical lognormal distribution [10]. In addition, due to the collocation of the on board antennae, possible obstructions affect equally all received signals. Thus, $\boldsymbol{\Xi}_{d}$ is a diagonal matrix composed of random elements that represent shadowing due to user mobility: $\boldsymbol{\Xi}_{d}=\operatorname{diag}\{\boldsymbol{\xi}\}$, where $\boldsymbol{\xi}=\left[\xi_{1}, \xi_{2} \ldots \xi_{n}\right]$. The probability density function of the random fading coefficients $\xi_{m}$ reads as

$$
p\left(\xi_{m}\right)=\frac{1}{\xi_{m} \sqrt{2 \pi \sigma_{m}^{2}}} \exp \left(-\frac{\left(\ln \xi_{m}-\mu_{m}\right)^{2}}{2 \sigma_{m}^{2}}\right), \quad \xi_{m} \geq 0 .
$$


where $\mu_{m}(\mathrm{~dB})$ and $\sigma_{m}(\mathrm{~dB})$ are the mean and standard deviation of the of variable's natural logarithm, respectively.

An important observation that simplifies the theoretical analysis is the transformation of (4) into a more tractable form. In particular, we notice that receive correlation imposes rank deficiency on the matrix $\tilde{\mathbf{H}}_{R}$. According to (8), $\tilde{\mathbf{H}}_{R}$ is a matrix with identical rows, as can be easily verified. Likewise, $\overline{\mathbf{H}}_{R}$ has by assumption identical rows: Each user has the same LoS component towards the receiver, since the distance between satellite antennae is infinitesimal compared to the user-satellite propagation path. The sum of unit-rank matrices with identical rows leads to a total channel matrix $\mathbf{H}_{R}$ with identical rows and thus unit rank: $\operatorname{rank}\left(\mathbf{H}_{R}\right)=1$. The Hadamard product between two matrices can be transformed into matrix product in the special case where the second is a unit rank matrix with identical rows. Then, the Hadamard product between the initial matrices is equivalent to the product between the first one and a diagonal matrix containing row elements of the unit-rank matrix ${ }^{2}$. Taking this observation into consideration, (4) can be transformed into

$$
\mathbf{Z}=\mathbf{B} \mathbf{H}_{d} \boldsymbol{\Xi}_{d}^{1 / 2}
$$

where $\mathbf{H}_{d}$ is a diagonal matrix containing i.i.d. non-zero mean complex circularly symmetric elements.

\section{CAPACITY ANALYSIS}

The considered system is a Multi-User Single Input Multiple Output (MU-SIMO) MAC system, whose capacity, under uniform power allocation, is defined as [3]

$$
C_{\text {erg }}=\mathcal{E}\left\{\log _{2} \operatorname{det}\left(\mathrm{I}_{n}+\gamma \mathbf{Z}^{\dagger} \mathbf{Z}\right)\right\} .
$$

The proposed model, assumes no coordination among transmit antennas, so every user transmits at the same $\operatorname{SNR}(\gamma)$, which represents the ratio of the power transmitted by every user over the equivalent noise power at the receiver.

\section{A. Lower bounds}

Following a similar line of reasoning as in [24] and [10], the ergodic capacity of a symmetric $n \times n$ MIMO system, can be lower bounded as

$$
C_{\text {erg }} \geqslant n \log _{2}\left(1+\gamma \exp \left(\frac{1}{n} \mathcal{E}\left\{\ln \left(\operatorname{det}\left(\mathbf{Z}^{\dagger} \mathbf{Z}\right)\right)\right\}\right)\right),
$$

with the use of Minkowski's inequality. Considering $\mathbf{Z}$ as in (10) and by exploiting the symmetrical nature of the system, (12) yields

$$
\begin{aligned}
& C_{e r g} \geqslant n \log _{2}\left(1+\gamma \exp \left(\frac { 1 } { n } \left(\mathcal{E}\left\{\ln \left(\operatorname{det}\left(\mathbf{B B}^{\dagger}\right)\right)\right\}\right.\right.\right. \\
&\left.\left.\left.+\mathcal{E}\left\{\ln \left(\operatorname{det} \boldsymbol{\Xi}_{d}\right)\right\}+\mathcal{E}\left\{\ln \left(\operatorname{det}\left|\mathbf{H}_{d}\right|^{2}\right)\right\}\right)\right)\right) .
\end{aligned}
$$

${ }^{2}$ In the channel derivations this property is used in more than one occasions [23]. It results from the assumption that channel coefficients between each user and every receive antenna are identical.
Since $\mathbf{B}$ is deterministic, the remaining non deterministic terms of (13) need to be calculated. To this end, we first exploit the fact that $\boldsymbol{\Xi}_{d}$ is diagonal, to obtain

$$
\mathcal{E}\left\{\ln \left(\operatorname{det} \boldsymbol{\Xi}_{d}\right)\right\}=\sum_{m=1}^{n} \mathcal{E}\left\{\ln \left(\xi_{m}\right)\right\}=\sum_{m=1}^{n} \mu_{m} .
$$

Finally, to deduce an analytical expression for the term $\mathcal{E}\left\{\ln \left(\operatorname{det}\left|\mathbf{H}_{d}\right|^{2}\right)\right\}$, the procedure described hereafter is necessary: The diagonal matrix $\left|\mathbf{H}_{d}\right|^{2}$ is composed of Rician elements, i.e. $\mathbf{H}_{d}=\operatorname{diag}\{\mathbf{h}\}$, where $\mathbf{h}=\left[h_{1}, h_{2} \ldots h_{n}\right]$. Each element of vector $\mathbf{h}$ is given by $h_{i}=\sqrt{\frac{K}{K+1}} \bar{h}_{i i}+\sqrt{\frac{1}{K+1}} \tilde{h}_{i i}$, where $\bar{h}$ is complex deterministic and $\tilde{h} \sim \mathcal{C N}(0,1)$, representing the LoS and the Rayleigh components of the signal respectively. Let us consider the following calculations:

$$
\begin{aligned}
& \mathcal{E}\left\{\ln \left(\operatorname{det}\left|\mathbf{H}_{d}\right|^{2}\right)\right\}= \\
& =\mathcal{E}\left\{\ln \left(\prod_{i=1}^{n}\left|\sqrt{\frac{K}{K+1}} \bar{h}_{i i}+\sqrt{\frac{1}{K+1}} \tilde{h}_{i i}\right|^{2}\right)\right\} \\
& =\sum_{i=1}^{n} \mathcal{E}\left\{\ln \left|\sqrt{\frac{K}{K+1}} \bar{h}_{i i}+\sqrt{\frac{1}{K+1}} \tilde{h}_{i i}\right|^{2}\right\} \\
& =-n \ln (K+1)+\sum_{i=1}^{n} \mathcal{E}\left\{\ln \left|\sqrt{K} \bar{h}_{i i}+\tilde{h}_{i i}\right|^{2}\right\} .
\end{aligned}
$$

Hence, the last term of (15) needs to be deduced. From [13], the elements of $\left|\mathbf{H}_{d}\right|^{2}$ follow a non-central chi-squared $\left(\chi^{2}\right)$ distribution. According to [25] and [26], the expectation of the natural logarithm of random variable following the $\chi^{2}$ distribution can be calculated as follows:

$$
\mathcal{E}\left\{\ln \sum_{j=1}^{l}\left|U_{j}+w_{j}\right|^{2}\right\}=g_{l}\left(s^{2}\right),
$$

where $U \sim \mathcal{C N}(0,1), w$ is a deterministic complex number, $2 l$ are the degrees of freedom of the $\chi^{2}$ distribution (i.e. the number of squared normal random variables summed $)^{3}$ and $s^{2}$ is the distribution's non-centrality parameter, given from

$$
s^{2}=\sum_{j=1}^{l}\left|w_{j}\right|^{2} .
$$

By examining (15), it is clear that $l=1$ complex channel coefficients are added, thus $\left|\mathbf{H}_{d}\right|^{2}$ follows a $\chi^{2}$-distribution with two degrees of freedom, (i.e. $\left|\mathbf{H}_{d}\right|^{2} \sim \chi_{2}^{2}\left(s^{2}\right)$ ). Function $g_{1}$ is defined as [26]:

$$
g_{1}\left(s^{2}\right) \triangleq \ln \left(s^{2}\right)-E i\left(-s^{2}\right),
$$

where $E i(x)$ denotes the exponential integral function, and $\psi(x)$ is Euler's digamma function. Hence, the last term of

\footnotetext{
${ }^{3}$ Of particular interest is the case of even number of degrees of freedom since not only it leads to closed form solutions but is also realistic. The squared norm of complex random variables distributed as $\mathcal{C N}(0,1)$ leads to
} an even number of total squared normal variables. 
(15) can be calculated using (16):

$$
\mathcal{E}\left\{\ln \left(\operatorname{det}\left|\mathbf{H}_{d}\right|^{2}\right)\right\}=-n \ln (K+1)+\sum_{i=1}^{n} g_{1}\left(s_{i}^{2}\right),
$$

where $s_{i}^{2}=\left|w_{i}\right|^{2}=K\left|\bar{h}_{i i}\right|^{2}$. Subsequently, the analytically deduced lower bound for a symmetrical $n \times n$ multibeam joint processing system operating in composite Rician/lognormal channel with correlated receive antennas reads as

$$
\begin{aligned}
C_{\text {erg }} & \geq n \log _{2}\left(1+\gamma \exp \left(\frac { 1 } { n } \left(\ln \left(\operatorname{det}\left(\mathbf{B} \cdot \mathbf{B}^{\dagger}\right)\right)\right.\right.\right. \\
& \left.\left.\left.+\sum_{k=1}^{n} \mu_{m}-n \ln (K+1)+\sum_{i=1}^{n} g_{1}\left(s_{i}^{2}\right)\right)\right)\right) .
\end{aligned}
$$

\section{B. Numerical results}

A set of Monte Carlo simulations is carried out to calculate the channel capacity, given by (11). The results are depicted in Fig. 1, over a wide range of SNR values. In the same figure, the analytically deduced lower bound is compared to the theoretical capacity. Finally, the achievable capacity of multibeam systems employing four color frequency reuse, over identical channel assumptions, given by

$$
C_{4 c}=\mathcal{E}\left\{\frac{1}{4} \sum_{i=1}^{n} \log _{2}\left(1+\frac{\left|z_{i i}\right|^{2}}{\sum_{j \neq i, j \in A_{C}}\left|z_{i j}\right|^{2}+(4 \gamma)^{-1}}\right)\right\},
$$

is plotted versus SNR in the same figure. The channel coefficients $z_{i j}$ are given in (2), $\gamma$ is defined in (13), while $A_{C}^{i}$ is the set of cochannel to the $i$-th, beams.

In the context of mathematical analysis, the behavior of the proposed bound in the extreme cases of low and high SNR is depicted in Fig. 1. Amid the lack of tightness in the low SNR region (see Fig. 1(b)), it is illustrated that the bound becomes exact for SNR values more than $30 \mathrm{~dB}$. The behavior of the deduced formula was also examined for variable Rician factors. The results are depicted in Fig. 2 for a specific SNR value (i.e. $S N R=25 d B$ ) versus a typical range of the Rician factor in a satellite channel [27]. According to these results, the bound remains exact with respect to the Rician factor and can thus be used for various satellite scenarios.

To the end of investigating the potential gains of multibeam joint decoding, the link budget of a satellite system for mobile users has been calculated in Table I. Referring to these calculations, a low SNR analysis is necessary in order to provide tighter bounds in the area of practical interest (i.e. [-5 - 25] dB). Furthermore, according to Fig. 1, the gain over a traditional frequency reuse system is more than threefold at the high SNR region and approximately twofold for lower SNR values. This result indicates that potential operation in higher SNR regions, will increase the gain of multibeam joint decoding techniques in a satellite communications network.

\section{CONCLUSIONS AND FUTURE WORK}

The capacity of a MU-SIMO composite Rician/lognormal satellite channel has been studied and analytically lower bounded. The proposed lower bound converges across the

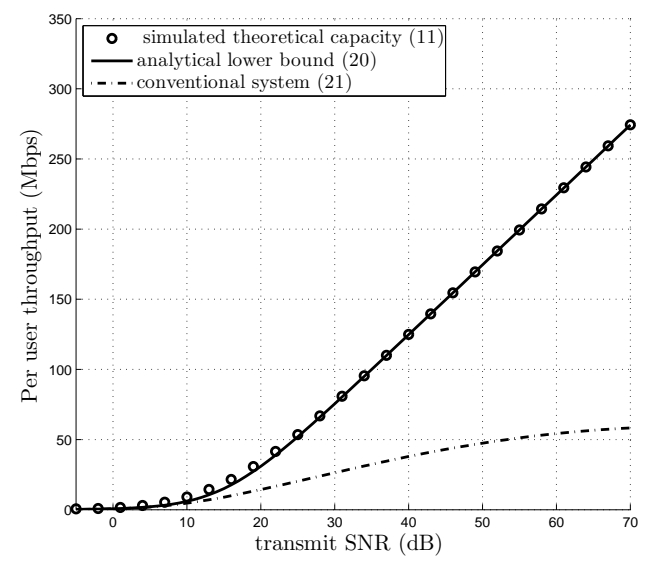

(a)

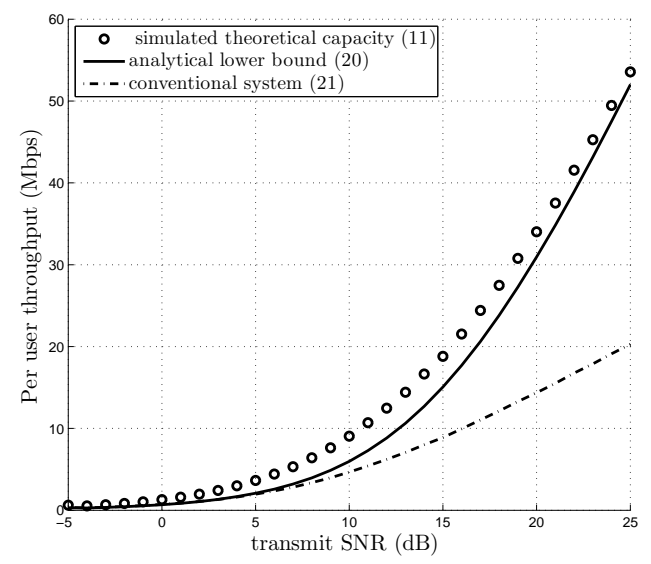

(b)

Fig. 1. Theoretical and analytical results versus a large range of transmit SNR compared to the capacity of a conventional system employing a four color frequency reuse scheme

entire SNR range and becomes exact at high SNRs. Additionally, the bound remains exact for a typical range of the Rician factor which is an important parameter of the system model. Subsequently, the presented analysis is suitable for multibeam SatCom systems, where multiple mobile users are transmitting and the received signals at the multibeam satellite antenna are jointly decoded by an ideal hyper receiver. The comparison with conventional frequency reuse schemes quantifies the significant gains of the proposed techniques. More than twofold increase in the user throughput is expected in the currently adopted SNR region of operation. Moreover, potential increase of the transmit SNR, can increase the throughput of the uplink of next generation SatComs more than three times, when conventional systems would be limited by the interbeam interferences.

Future extensions of this work include the exploration of multibeam joint decoding techniques under more realistic receiver architectures, e.g. linear minimum mean-square error (LMMSE), as well as a low SNR analysis. 


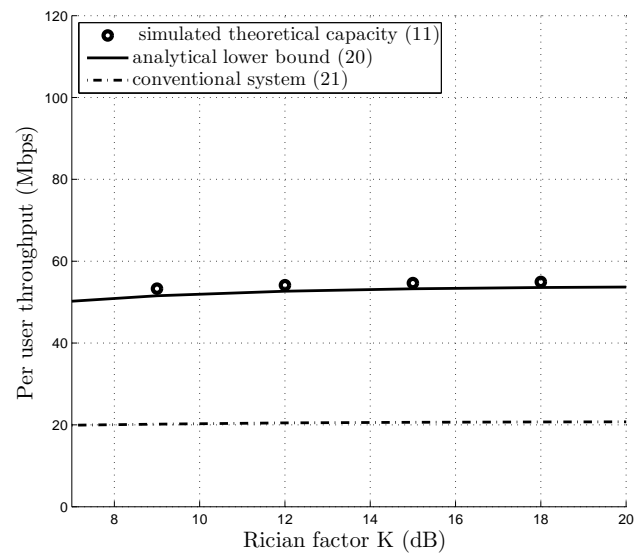

Fig. 2. Theoretical and analytical results versus a typical, for a satellite scenario, range of the Rician factor, with $S N R=25 d B$, compared to the reference conventional system

\section{TABLE I}

LinK BUdget \& Simulation PARAMETERS

\begin{tabular}{l|c} 
Parameter & Value \\
\hline Frequency Band & S $(2.2 \mathrm{GHz})$ \\
User Link Bandwidth $B_{u}$ & $15 \mathrm{MHz}$ \\
Mobile terminal RF power & {$[-3-24.5] \mathrm{dBW}$} \\
Receiver noise power $N$ & $-133 \mathrm{dBW}$ \\
Free Space Loss $L$ & $190 \mathrm{~dB}$ \\
Atmospheric Loss & $0.5 \mathrm{~dB}$ \\
Polarization Loss & $3 \mathrm{~dB}$ \\
Mobile Antenna Gain $G_{T}$ & $3 \mathrm{~dB}$ \\
Max satellite antenna gain $G_{R}$ & $52 \mathrm{dBi}$ \\
Fading Margin & $3 \mathrm{~dB}$ \\
Transmit SNR & {$[-5-25] \mathrm{dB}$} \\
\hline Beam Radius & $350 \mathrm{~km}$ \\
Area Radius & $1000 \mathrm{~km}$ \\
Number of Beams $n$ & 7 \\
Rician factor $K$ & $13 \mathrm{~dB}$ \\
Lognormal Shadowing $\mu_{m}, \sigma_{m}$ & $-2.62 \mathrm{~dB}, 1.6 \mathrm{~dB}$ \\
Iterations & 1000
\end{tabular}

\section{ACKNOWLEDGMENT}

This work was partially supported by the National Research Fund, Luxembourg under the project " $C O^{2} S A T$ : Cooperative \& Cognitive Architectures for Satellite Networks" and by the Satellite Network of Excellence (SatNEx3). The work of M. Matthaiou has been supported in part by the Swedish Governmental Agency for Innovation Systems (VINNOVA) within the VINN Excellence Center Chase.

\section{REFERENCES}

[1] D. Mignolo, R. Emiliano, A. Ginesi, A. B. Alamanac, P. Angeletti, and M. Harverson, "Approaching terabit/s satellite capacity: A system analysis," in Proc. Ka Broadband Conf., Oct. 2011.

[2] 3GPP, "Further advancements for E-UTRA physical layer aspects," 3rd Generation Partnership Project, Tech. Rep. TR 36.814.

[3] I. E. Telatar, "Capacity of multi-antenna Gaussian channels," Eur. Trans. Telecommun., vol. 10, no. 6, pp. 585-595, Nov. 1999.

[4] S. V. Hanly and P. A. Whiting, "Information-theoretic capacity of multireceiver networks," Telecommun. Syst., vol. 1, pp. 1-42, 1993.

[5] S. Chatzinotas, M. Imran, and R. Hoshyar, "On the multicell processing capacity of the cellular MIMO uplink channel in correlated Rayleigh fading environment," IEEE Trans. Wireless Commun., vol. 8, no. 7, pp. 3704-3715, July 2009.
[6] S. Chatzinotas, M. Imran, and C. Tzaras, "On the capacity of variable density cellular systems under multicell decoding," IEEE Commun. Lett., vol. 12 , no. 7, pp. 496-498, July 2008.

[7] O. Somekh and S. Shamai, "Shannon-theoretic approach to a Gaussian cellular multiple-access channel with fading," IEEE Trans. Inf. Theory, vol. 46, no. 4, pp. 1401-1425, July 2000.

[8] J. Hoydis, A. Kammoun, J. Najim, and M. Debbah, "Outage performance of cooperative small-cell systems under Rician fading channels," in Proc. IEEE Int. Work. Signal Process. Advances Wireless Commun. (SPAWC), June 2011, pp. 551-555.

[9] D. Kaltakis, M. Imran, and C. Tzaras, "Information theoretic capacity of cellular multiple access channel with shadow fading," IEEE Trans. Commun., vol. 58, no. 5, pp. 1468-1476, May 2010.

[10] M. Matthaiou, N. D. Chatzidiamantis, and G. K. Karagiannidis, "A new lower bound on the ergodic capacity of distributed MIMO systems," IEEE Signal Process. Lett., vol. 18, no. 4, pp. 227-230, Apr. 2011.

[11] M. Matthaiou, N. Chatzidiamantis, G. Karagiannidis, and J. Nossek, "On the capacity of generalized- $K$ fading MIMO channels," IEEE Trans. Signal Process., vol. 58, no. 11, pp. 5939-5944, Nov. 2010.

[12] C. Zhong, K.-K. Wong, and S. Jin, "Capacity bounds for MIMO nakagami- $m$ fading channels," IEEE Trans. Signal Process., vol. 57, no. 9, pp. 3613-3623, Sept. 2009.

[13] N. Letzepis and A. Grant, "Capacity of the multiple spot beam satellite channel with Rician fading," IEEE Trans. Inf. Theory, vol. 54, no. 11 pp. 5210-5222, Nov. 2008.

[14] S. Jin, X. Gao, and X. You, "On the ergodic capacity of rank-1 Riceanfading MIMO channels," IEEE Trans. Inf. Theory, vol. 53, no. 2, pp. 502-517, Feb. 2007.

[15] S. Al-Ahmadi and H. Yanikomeroglu, "The ergodic and outage capacities of distributed antenna systems in generalized- $k$ fading channels," in Proc. IEEE Int. Symp. Personal Indoor Mob. Radio Commun. (PIMRC), Sept. 2010, pp. $662-666$.

[16] G. Alfano, A. De Maio, and A. M. Tulino, "A theoretical framework for LMS MIMO communication systems performance analysis," IEEE Trans. Inf. Theory, vol. 56, no. 11, pp. 5614-5630, Nov. 2010.

[17] M. Moher, "Multiuser decoding for multibeam systems," IEEE Trans. Veh. Technol., vol. 49, no. 4, pp. 1226-1234, Jul. 2000.

[18] G. Zheng, S. Chatzinotas, and B. Ottersten, "Generic Optimization of Linear Precoding in Multibeam Satellite Systems," ArXiv e-prints, Sep. 2011.

[19] S. Chatzinotas, G. Zheng, and B. Ottersten, "Energy-efficient mmse beamforming and power allocationin multibeam satellite systems," in Asilomar Conference on Signals, Systems and Computers (ACSSC'11), Nov 2011.

[20] _ - "Joint precoding with flexible power constraints in multibeam satellite systems," in IEEE Global Telecommunications Conference (GLOBECOM 2011), Houston, Texas, 2011.

[21] M. Diaz, N. Courville, C. Mosquera, G. Liva, and G. Corazza, "Nonlinear interference mitigation for broadband multimedia satellite systems," in Proc. Int. Work. Sat. Space Commun. (IWSSC), Sept. 2007, pp. $61-65$

[22] A. M. Tulino and S. Verdú, "Random matrix theory and wireless communications," Commun. Inf. Theory, vol. 1, no. 1, pp. 1-182, 2004.

[23] N. Letzepis and A. Grant, "Information capacity of multiple spot beam satellite channels," in Proc. Australian Commun. Th. Work., Feb. 2005, pp. $168-174$.

[24] O. Oyman, R. Nabar, H. Bölcskei, and A. Paulraj, "Characterizing the statistical properties of mutual information in MIMO channels," IEEE Trans. Signal Process., vol. 51, no. 11, pp. 2784 - 2795, Nov. 2003.

[25] A. Lapidoth and S. Moser, "Capacity bounds via duality with applications to multiple-antenna systems on flat-fading channels," IEEE Trans. Inf. Theory, vol. 49, no. 10, pp. 2426-2467, Oct. 2003

[26] S. Moser, "Duality-based bounds on channel capacity," Ph.D. dissertation, Swiss Federal Institute of Technology (ETH), Switzerland, Oct. 2004.

[27] B. Vucetic and J. Du, "Channel modeling and simulation in satellite mobile communication systems," IEEE J. Select. Areas Commun., vol. 10, no. 8, pp. 1209-1217, Oct. 1992. 